\title{
Change in the cross-sectional area of the thecal sac following balloon kyphoplasty for pathological vertebral compression fractures prior to spine stereotactic radiosurgery
}

\author{
Eric Lis, MD, ${ }^{1}$ llya Laufer, MD, ${ }^{2}$ Ori Barzilai, MD, ${ }^{2}$ Yoshiya Yamada, MD, ${ }^{3}$ Sasan Karimi, MD, ${ }^{1}$ \\ Lily McLaughlin, BS, ${ }^{2}$ George Krol, MD, ${ }^{1}$ and Mark H. Bilsky, MD ${ }^{2}$
}

\begin{abstract}
1Department of Radiology, Neuroradiology Service; and Departments of ${ }^{2}$ Neurosurgery and ${ }^{3}$ Radiation Oncology, Memorial Sloan Kettering Cancer Center, New York, New York
\end{abstract}

\begin{abstract}
OBJECTIVE Percutaneous vertebral augmentation procedures such as vertebroplasty and kyphoplasty are often performed in cancer patients to relieve mechanical axial-load pain due to pathological collapse deformities. The collapsed vertebrae in these patients can be associated with varying degrees of spinal canal compromise that can be worsened by kyphoplasty. In this study the authors evaluated changes to the spinal canal, in particular the cross-sectional area of the thecal sac, following balloon kyphoplasty (BKP) prior to stereotactic radiosurgery (SRS).

METHODS The authors retrospectively reviewed the records of all patients with symptomatic vertebral compression fractures caused by metastatic disease who underwent kyphoplasty prior to single-fraction SRS. The pre-BKP crosssectional image, usually MRI, was compared to the post-BKP CT myelogram required for radiation treatment planning. The cross-sectional area of the thecal sac was calculated pre- and postkyphoplasty, and intraprocedural CT imaging was reviewed for epidural displacement of bone fragments, tumor, or polymethylmethacrylate (PMMA) extravasation. The postkyphoplasty imaging was also evaluated for evidence of fracture progression or fracture reduction.
\end{abstract}

RESULTS Among 30 consecutive patients, 41 vertebral levels were treated with kyphoplasty, and 24\% (10/41) of the augmented levels showed a decreased cross-sectional area of the thecal sac. All 10 of these vertebral levels had preexisting epidural disease and destruction of the posterior vertebral body cortex. No bone fragments were displaced posteriorly. Minor epidural PMMA extravasation occurred in 20\% (8/41) of the augmented levels but was present in only 1 of the 10 vertebral segments that showed a decreased cross-sectional area of the thecal sac postkyphoplasty.

CONCLUSIONS In patients with preexisting epidural disease and destruction of the posterior vertebral body cortex who are undergoing BKP for pathological fractures, there is an increased risk of further mass effect upon the thecal sac and the potential to alter the SRS treatment planning.

https://thejns.org/doi/abs/10.3171/2018.6.SPINE18206

KEYWORDS kyphoplasty; spine metastasis; epidural disease; oncology

$\mathrm{T}$ HE majority of patients who die from cancer have skeletal metastases, most commonly to the spine, with spine metastasis present in approximately $30 \%$ of oncological patients. ${ }^{19}$ Pain symptoms related to spine metastases are often multifactorial and may include tumor-associated biological pain, radiculopathy, and mechanical instability. ${ }^{15}$ Vertebral compression fractures of- ten occur as a consequence of metastatic disease to the spine and are a source of significant morbidity, often resulting in mechanical instability described as axial-load or movement-related pain. Balloon kyphoplasty (BKP) is a minimally invasive procedure that can stabilize a pathological vertebral compression fracture, offering patients decreased pain, increased mobility, and an overall im-

ABBREVIATIONS BKP = balloon kyphoplasty; ESCCS = epidural spinal cord compression scale; $\mathrm{PMMA}=$ polymethylmethacrylate; $\mathrm{ROI}=$ region of interest; $\mathrm{SSRS}=$ spine stereotactic radiosurgery; VAS = visual analog scale.

SUBMITTED February 22, 2018. ACCEPTED June 13, 2018.

INCLUDE WHEN CITING Published online October 19, 2018; DOI: 10.3171/2018.6.SPINE18206. 
proved quality of life. 1,4,9,13 The Cancer Patient Fracture Evaluation (CAFE) provided class I evidence that percutaneous cement augmentation offers meaningful, durable palliation in this very complicated cancer population. ${ }^{4}$ Spine stereotactic radiosurgery (SSRS) can deliver an ablative radiation dose to the tumor while sparing adjacent critical structures, most importantly the spinal cord and cauda equina. The procedure is becoming more common as there is increasing evidence that it provides durable tumor control, overcoming the resistance often encountered with conventional external beam radiation. $3,11,14,15,17$ Treating the compression fracture prior to SSRS can facilitate the radiotherapy by providing pain relief that eases transfer to the treatment table and improves tolerance in the immobilization frame. However, concerns have arisen regarding the effect of the procedure on the spinal canal in the setting of vertebral metastases. ${ }^{6,8}$

Metastatic disease to the vertebrae often results in lytic destruction, possibly compromising the integrity of the posterior vertebral cortex. Additionally, epidural disease, with or without posterior vertebral cortex destruction, can also occur and result in varying degrees of spinal canal compromise. During BKP, bone tamps are inflated in the collapsed vertebral body to create a cavity for the polymethylmethacrylate (PMMA) to fill. Inflation of the bone tamps can result in the mechanical displacement of tumor and/or bone fragments into the spinal canal, potentially worsening or causing mass effect upon the thecal sac and resulting in symptomatic spinal cord, cauda equina, or nerve root compression. Even without symptomatic progression, high-grade spinal cord or cauda equina compression is a contraindication to SSRS; thus, kyphoplasty can reduce the ability to deliver a cytotoxic radiation dose within spinal cord or cauda equina constraints. ${ }^{6,8}$ The current study specifically examined the effects of BKP on the cross-sectional area of the thecal sac in patients with spine metastases and pathological compression fractures to assess the risk of neurological progression or increased spinal canal compromise prohibiting the delivery of SSRS.

\section{Methods \\ Patients}

Institutional review board approval was obtained for this study. We retrospectively reviewed the records of all patients with spine metastasis and symptomatic pathological vertebral compression fractures who had been referred for BKP for pain palliation prior to undergoing SSRS. All patients had been evaluated by a multidisciplinary spine team consisting of neuroradiologists (E.L., G.K.), neurosurgeons (M.H.B., I.L.), and a radiation therapist (Y.Y.). None of the patients had mechanical radiculopathy, myelopathy, or high-grade spinal cord or cauda equina compression, and all were deemed acceptable candidates for the procedure. The visual analog scale (VAS) scores for pain were recorded at the time of the initial clinic visit and at the approximate 4-week follow-up visit.

All patients underwent pre-BKP cross-sectional spine imaging usually consisting of MRI (29 patients), although one patient had pre-BKP contrast-enhanced CT. The BKP procedures were performed according to established in- stitutional guidelines with the patient under general anesthesia in the interventional radiology department on a GE Innova CT/angiography system with C-arm fluoroscopy and CT capabilities. ${ }^{10,21}$ All BKP procedures were performed with the Kyphon Express kit (Medtronic Spine) utilizing either a bilateral transpedicular or a parapedicular approach and 10- or 15-mm inflatable bone tamps. In all patients, the bone tamps were placed in the anterior two-thirds of the vertebral body. Biopsies were obtained in all patients. Intraprocedural CT scanning with the bone tamps inflated and immediate postkyphoplasty CT scanning were performed. At a later date, all patients underwent post-BKP CT myelography as part of the treatment planning for SSRS.

\section{Pre-BKP Analysis}

An axial image at the intended kyphoplasty level that best demonstrated the greatest degree of thecal sac and spinal canal compromise was selected. The cross-sectional area of the thecal sac on the selected axial image was determined by drawing a region of interest (ROI) around the thecal sac on a GE-PACS workstation, which then automatically calculated the cross-sectional area of the thecal sac (Figs. 1B and 2A). The greatest percentage loss of vertebral body height was also calculated by obtaining measurements from sagittal images at the plane of maximum loss of vertebral body height. Prefracture vertebral body height was determined by measuring the height of the unfractured component of the vertebral body; if the vertebral body was uniformly collapsed, the prefracture height was estimated by measuring the vertebral body heights of the adjacent unfractured levels. ${ }^{7,16}$ The ROIs and percentage vertebral body collapse were determined by a CAQ (certificate of added qualification) neuroradiologist (E.L.) and confirmed by a second CAQ neuroradiologist (S.K.).

Pre-BKP imaging studies (MRI, CT) of the levels that underwent BKP were also analyzed for the appearance of metastatic disease (i.e., lytic, sclerotic, or mixed), integrity of the posterior vertebral body cortex, and presence of epidural disease. Epidural disease at the level of the spinal cord was characterized according to the epidural spinal cord compression scale (ESCCS), ${ }^{5}$ and epidural disease in the lumbar spine below the conus medullaris was characterized as causing mild to moderate central spinal canal compromise.

\section{Intraprocedural Analysis}

The intraprocedural fluoroscopic and CT images were reviewed to assess the underlying metastatic disease as lytic, sclerotic, or mixed lytic-sclerotic in the patients who did not have a preprocedural CT scan. Also, the integrity of the posterior vertebral body cortex was recorded as either intact or not intact. Extravertebral PMMA extravasation with involvement of the epidural space was noted as well.

\section{Post-BKP CT Myelography Analysis}

The post-BKP CT myelogram was analyzed in a manner similar to that used for the prekyphoplasty cross- 


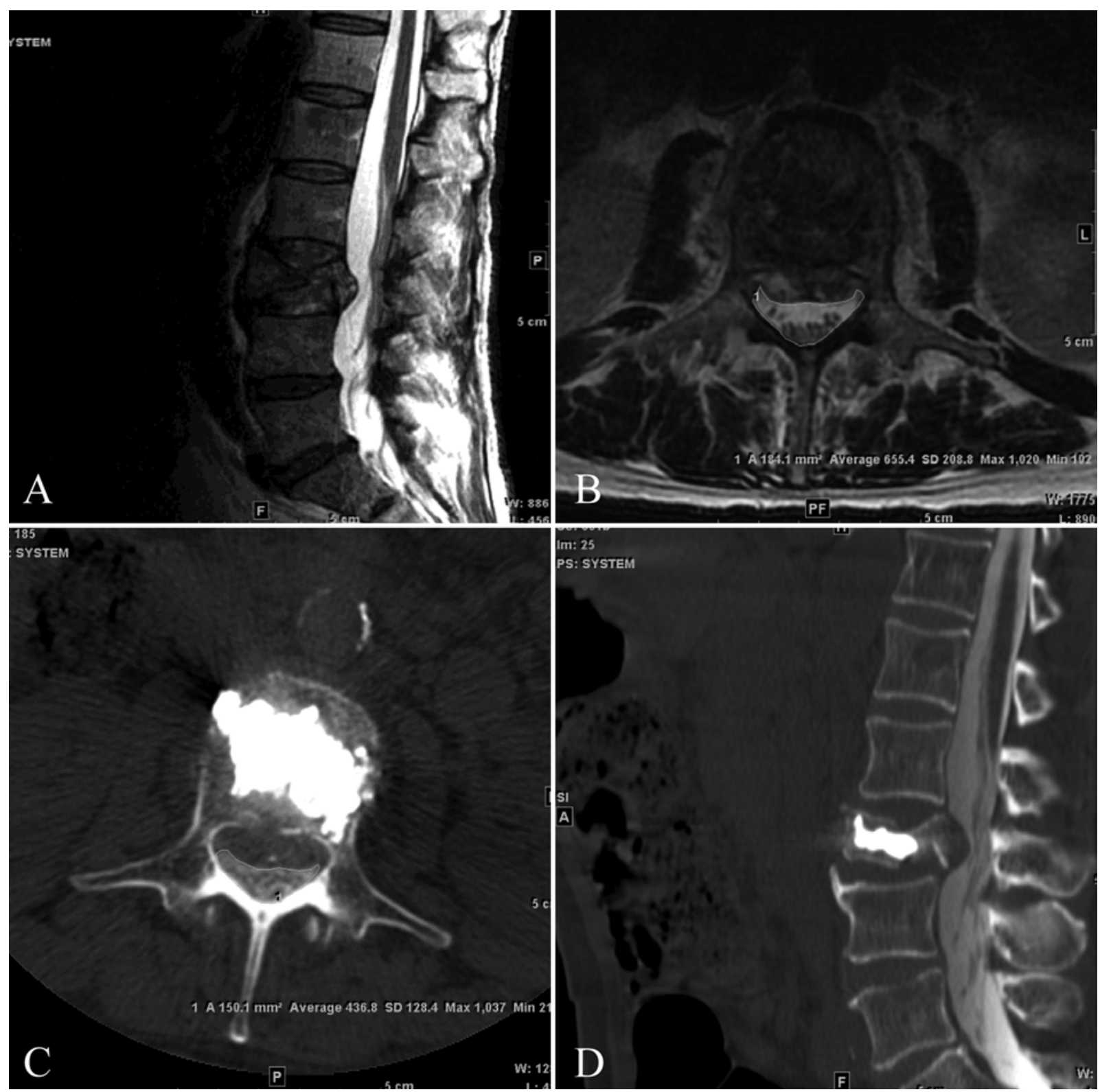

FIG. 1. Images obtained in a 74-year-old man with stage IV esophageal cancer and an L3 metastasis with pathological collapse. A: Pre-BKP sagittal T2-weighted MR image obtained through the lumbar spine, showing a pathological collapse of L3 with the mid vertebral body having lost about $67 \%$ of its estimated height. B: Pre-KBP axial T2-weighted image obtained through L3, revealing ventral epidural disease resulting in moderate spinal compromise. The cross-sectional area of the thecal sac, as outlined by the $\mathrm{ROI}$, was calculated to be $1.8 \mathrm{~cm}^{2}$. C: Post-BKP axial CT myelogram obtained through L3, showing destruction of the posterior vertebral cortex. The ROI outlining the thecal sac is also shown, with the cross-sectional area of the thecal sac calculated to be $1.5 \mathrm{~cm}^{2}$, a decrease of $17 \%$ compared to the pre-KBP cross-sectional area. D: Post-BKP sagittal CT myelogram showing fracture reduction, an increase of $57 \%$ in the midportion of the vertebral body compared to that in panel A. Increased epidural mass effect on the ventral thecal sac was confirmed. The time between the pre-BKP MR image and the CT myelogram was 10 days.

sectional imaging. An axial image was selected to best match the axial image from the pre-BKP image analysis. If needed, the post-BKP CT myelograms were reformatted to best approximate the angle and level of the axial image to the pre-BKP image. The cross-sectional area of the thecal sac at the kyphoplasty level was determined by first selecting a post-BKP axial CT myelogram and drawing a ROI outlining the thecal sac on a PACS workstation (Figs. $1 \mathrm{C}$ and $2 \mathrm{C}$ ). The percentage degree of vertebral body col- lapse at the kyphoplasty level was also acquired from the sagittal reformatted images.

\section{Results}

Balloon kyphoplasty was safely performed at $41 \mathrm{lev}-$ els in 30 consecutive patients -22 males, 8 females, mean age 60 years, age range $35-81$ years-who had metastatic disease to the thoracic and lumbar spine. The procedure 

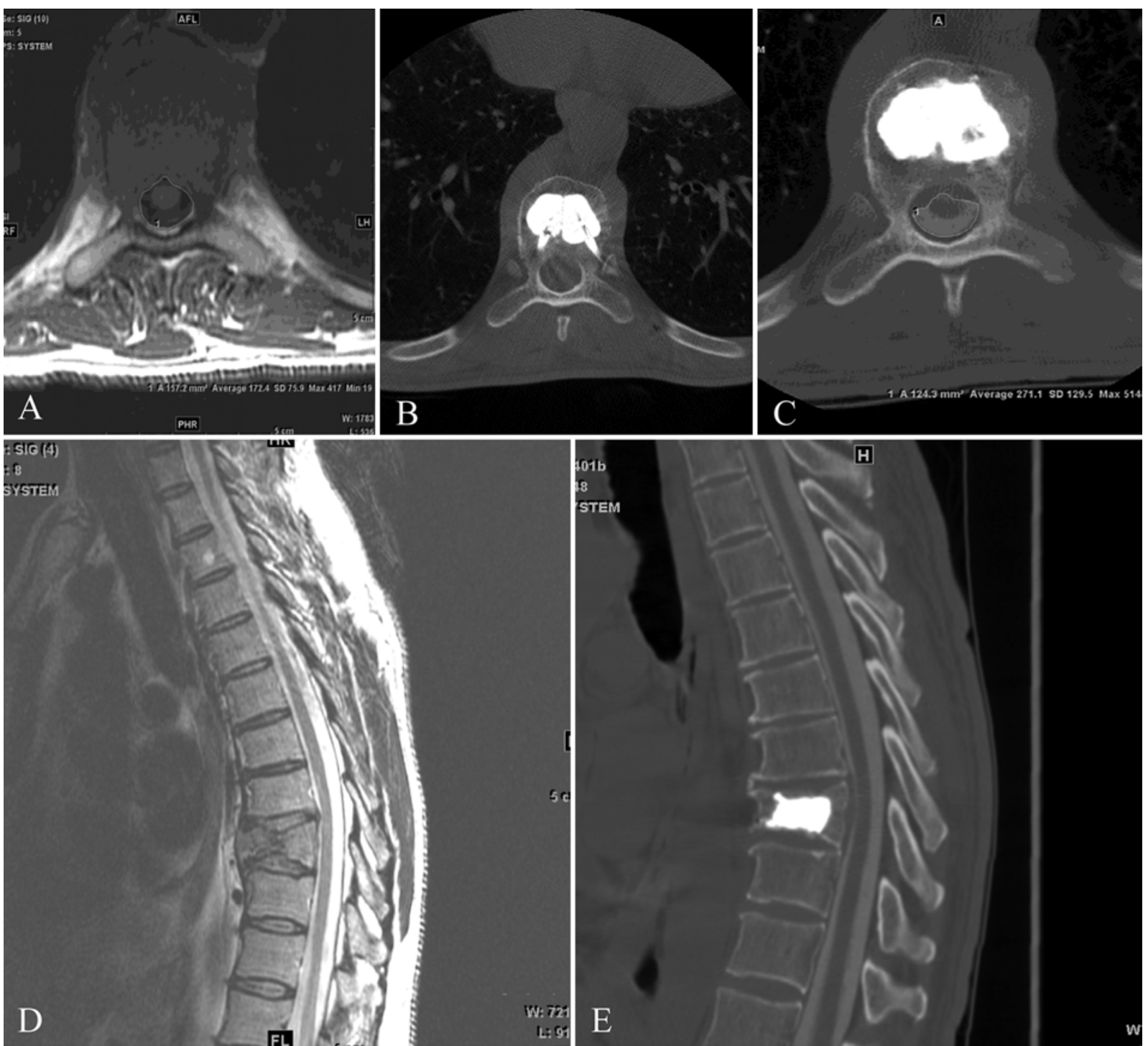

FIG. 2. Images obtained in a 54-year-old man with colon cancer and a T9 metastasis with pathological collapse. A: Pre-BKP axial T1-weighted MR image obtained through T9, showing mild ventral epidural disease (ESCCS grade 1b) and the ROI outlining the thecal sac, whose cross-sectional area was calculated to be $1.6 \mathrm{~cm}^{2}$. B: Axial CT image obtained through T9 at the time of the kyphoplasty, showing the position of the inflated bilateral $15 \mathrm{~mm}$ bone tamps with permeative destruction of the posterior vertebral cortex. C: Post-BKP axial CT obtained through T9 with the ROI outlining the thecal sac and showing a $25 \%$ decrease in the crosssectional area of the thecal sac, which now measures $1.2 \mathrm{~cm}^{2}$ (ESCCS grade 1c). D: Pre-BKP sagittal T2-weighted MR image showing a pathological collapse of T9 with an approximately $52 \%$ loss of vertebral body height at the mid vertebral body. E: PostBKP sagittal CT myelogram showing fracture reduction, an increase of $27 \%$ in the midportion of the vertebral body compared to that in panel D. Increased epidural soft tissue can also be identified. The time between pre-BKP MRI and the CT myelogram was 20 days.

was fairly evenly divided between the thoracic and lumbar spine: 21 and 20 levels, respectively. The average number of days between BKP and the post-BKP CT myelogram was 2.7 days (range 1-12 days). The average number of days between the initial pre-BKP spine MR image (CT) and the post-BKP CT myelogram was 17.5 days (range $3-63$ days).

\section{Pre-BKP and Intraprocedural Analysis}

The most common diagnoses obtained on the biopsies performed at the time of BKP were metastatic colon cancer (20\%), non-small cell lung cancer (20\%), and renal cell carcinoma (17\%). The imaging (CT) appearance of the metastatic lesions was found to be predominantly lytic $(59 \%)$ or mixed lytic-sclerotic (34\%; Table 1). The posterior vertebral body cortex was compromised in 54\% (22/41) of vertebral segments, with 78\% (32/41) of the vertebrae having some degree of epidural disease (Table 2). However, no patients were found to have high-grade spinal canal compromise or ESCCS grade > 1c, defined as spinal cord abutment without compression. The average loss of vertebral body height on the pre-BKP imaging was $31 \%$ 
TABLE 1. CT characterization of the vertebral metastasis

\begin{tabular}{cc}
\hline CT Appearance & Value \\
\hline Lytic & $59 \%(24 / 41)$ \\
\hline Sclerotic & $5 \%(2 / 41)$ \\
\hline Mixed & $34 \%(14 / 41)$ \\
\hline Normal & $2 \%(1 / 41)$ \\
\hline
\end{tabular}

(range 5\%-67\%; Table 3). The average amount of PMMA infused into each vertebral body during the BKP was 4.1 $\mathrm{ml}$ (range 1.3-7.2 ml).

\section{Post-BKP}

On the post-BKP CT myelogram, 24\% (10/41) of the augmented vertebral levels showed a decreased cross-sectional area of the thecal sac as compared to its appearance on pre-BKP spine imaging. The average decrease in the cross-sectional area of the thecal sac in this group was 21\% (range 15\%-30\%; Table 3). All 10 vertebral levels that showed a decreased cross-sectional area post-BKP were associated with preexisting epidural disease and destruction of the posterior vertebral body cortex (Figs. 1 and 2).

Some degree of fracture reduction was evident in $27 \%$ (11/41) of the augmented levels, whereas fracture progression was observed in 10\% (4/41) of the augmented levels.

Asymptomatic extravertebral cement extravasation was observed in 49\% (20/41) of the augmented levels with no extravasated cement extending beyond the treated level. Minor ventral epidural PMMA extravasation was encountered at 8 levels (20\%); however, only 1 treated level (L1) in this group was associated with a decreased cross-sectional area of the thecal sac post-BKP.

No bone fragments were displaced into the spinal canal, and no patient developed any neurological sequela related to the procedure. All patients underwent their planned SSRS.

Nearly all patients $(96 \%)$ reported at least some degree of improvement in their movement-related back pain. The VAS score showed an average improvement from 7.3 preBKP to 3.3 post-BKP.

\section{Discussion}

The benefit of BKP in the treatment of painful neoplastic vertebral compression fractures is well established in the literature. ${ }^{1,49}$ Kyphoplasty is often performed before SSRS, with the kyphoplasty treating the mechanical pain and the radiation therapy addressing the underlying tumor, providing local control, and treating the biological pain. ${ }^{12}$ In this paper, we describe the effects of BKP on the cross-sectional area of the thecal sac in patients with symptomatic pathological fractures, prior to being treated with stereotactic radiation therapy.

In this group of patients who underwent BKP, the only augmented vertebral levels that showed a decrease in the cross-sectional area of the thecal sac were those that had preexisting epidural disease and compromise of the posterior vertebral body cortex. The likely mechanism behind this was inflation of the bone tamps, which compresses
TABLE 2. Integrity of posterior vertebral body cortex and the presence of epidural disease prekyphoplasty

\begin{tabular}{cc}
\hline Parameter & Value \\
\hline Pst vertebral body cortex & \\
\hline Intact & $46 \%(19 / 41)$ \\
\hline Compromised & $54 \%(22 / 41)$ \\
\hline Epidural disease & $78 \%(32 / 41)$ \\
\hline
\end{tabular}

Pst = posterior.

and displaces tissue within the vertebral body. ${ }^{6}$ Tumor displaced by inflation of the bone tamps will most likely be directed toward the path of least resistance, regions where the cortex is not intact. If the posterior cortex is compromised, tumor may be displaced into the ventral epidural space, which represents the path of least resistance. We think this displacement of tumor by the bone tamps and its maintenance by the infused PMMA account for the decreased cross-sectional area of the thecal sac, as demonstrated in both Figs. 1 and 2. Close inspection of Fig. 2C shows segmental permeative destruction of the anterolateral walls of the vertebral body, especially on the left, as well as the posterior cortex. The post-BKP CT myelogram compared to the preprocedural MR image for this patient showed not only increased ventral epidural tumor but also increased paraspinal tumor, which indicates that there was tumor displacement in all directions about the vertebral body. Thus, vertebrae with only the posterior cortex disrupted may be at even greater risk for canal compromise from displaced tumor because the pressure associated with inflation of the bone tamps may only be dissipated posteriorly. In addition, we suspect that tumor compression or compaction was occurring, which would explain why there was less tumor displacement than one might expect given the volume of PMMA infused into each of the vertebrae. In our institutional experience, kyphoplasty is indicated over vertebroplasty in the neoplastic setting principally because the PMMA can be placed under low pressure, decreasing the risk of extravasation. We use larger volumes of PMMA than those described in the osteoporotic literature in order to prevent fracture progression.

In this study just over $25 \%$ of the augmented levels showed evidence of at least some degree of height restoration. More interestingly, 60\% (6/10) of the levels that showed increased spinal canal compromise post-BKP also had some degree of height restoration. The increased spinal canal compromise in these vertebrae post-BKP may in part reflect more aggressive inflation of the bone tamps, although the plasticity of a tumor-replaced vertebrae also contributes to height restoration.

Our findings support the notion that the presence of epidural disease and destruction of the posterior vertebral body cortex are not absolute contraindications to BKP, although one must recognize that further compromise of the epidural space can occur. The importance of this is two-fold. First, kyphoplasty has the potential to acutely increase the epidural disease, causing frank spinal cord compression requiring immediate surgical decompression. ${ }^{8}$ Second, the procedure can disqualify a patient for SSRS if there were an increase in spinal canal compromise to 
TABLE 3. Summary of levels that showed a decreased cross-sectional area of the thecal sac post-BKP

\begin{tabular}{clcccccc}
\hline $\begin{array}{c}\text { Level } \\
\text { Treated }\end{array}$ & $\begin{array}{c}\text { Primary } \\
\text { Cancer }\end{array}$ & Appearance & $\begin{array}{c}\text { Max Loss in } \\
\text { VB Height }\end{array}$ & $\begin{array}{c}\text { Cement } \\
\text { Vol }(\mathrm{ml})\end{array}$ & $\begin{array}{c}\text { Epidural Cement } \\
\text { Extravasation }\end{array}$ & $\begin{array}{c}\text { Fracture Progression } \\
\text { or Reduction }\end{array}$ & $\begin{array}{c}\text { Change in Area } \\
\text { of Thecal Sac }\end{array}$ \\
\hline T12 & Colon & Lytic & $40 \%$ & 3.5 & No & NC & $-17 \%$ \\
\hline L1 & Renal & Lytic & $5 \%$ & 3.5 & Minimal & NC & $-24 \%$ \\
\hline T9 & Colon & Mixed & $52 \%$ & 7.2 & No & FR 27\% & $-25 \%$ \\
\hline L3 & Esophageal & Mixed & $67 \%$ & 6.0 & No & FR 57\% & $-17 \%$ \\
\hline L3 & Adrenal & Lytic & $50 \%$ & 3.2 & No & NC & $-22 \%$ \\
\hline T9 & Renal & Lytic & $24 \%$ & 4.8 & No & FR 25\% & $-30 \%$ \\
\hline T5 & Thymus & Mixed & $53 \%$ & 2.4 & No & FR 44\% & $-20 \%$ \\
\hline L1 & Renal & Lytic & $48 \%$ & 4.8 & No & FR 38\% & $-15 \%$ \\
\hline L2 & Renal & Lytic & $25 \%$ & 4.8 & No & FR 10\% & $-21 \%$ \\
\hline L1 & Renal & Lytic & $40 \%$ & 6.0 & No & NC & $-17 \%$ \\
\hline
\end{tabular}

$\mathrm{FR}=$ fracture reduction; $\mathrm{NC}=$ no change; $\mathrm{VB}=$ vertebral body.

Posterior cortex was not intact at any treated level.

the point that high-dose radiation could no longer be given because of spinal cord and cauda equina dose constraints. With these two points in mind, one must obtain an MR image of the spine within a relatively short time before the kyphoplasty procedure to adequately assess the degree of spinal canal compromise that may have occurred due to tumor progression or further vertebral body collapse. Recent imaging is important in identifying a neurologically asymptomatic patient who has developed increased epidural disease and potentially high-grade epidural spinal cord compression and is at higher risk for incurring a neurological injury from tumor displacement via BKP.

Attention to proper technique, from patient positioning to fluoroscopy, is of the utmost importance to ensure that true anteroposterior and lateral views are being obtained and that the expected position of the posterior margin of the vertebral body can be determined. Needle trajectory is important to ensure a transpedicular approach that avoids breeches of the medial cortex of the pedicle. An extrapedicular approach may be of benefit when there is preexisting lateral epidural disease. Placement of bone tamps in the middle to anterior third of the vertebral body is ideal, avoiding the posterior aspect of the vertebral body as much as possible. Slow inflation of the bone tamps under lateral fluoroscopy is needed to make sure they do not quickly pull back toward the spinal canal into the region of dominant lytic change and to allow the pressure associated with inflation of the bone tamps to be dissipated slowly and ideally in all directions.

Meticulous attention is required during infusion of the PMMA. The PMMA needs to be of an appropriate viscosity and should be slowly and intermittently infused into the cavities created while imaging in the lateral fluoroscopic projection. Infusion is terminated when it extends preferentially into the posterior third of the vertebral body or close to the epidural space or neural foramina. The goal is to find the balance between getting enough of the PMMA into the vertebral body to provide stability and limit fracture progression while avoiding any further significant compromise of the spinal canal or significant PMMA extravasation. Of the 10 patients who had increased spinal canal compromise following BKP, only one had associ- ated ventral epidural cement extravasation, although it was minimal and without significant additional displacement of the thecal sac.

All patients in this study did undergo their scheduled SSRS following the kyphoplasty. That said, however, a relative contraindication to BKP prior to SSRS would be a destroyed posterior vertebral body cortex and epidural disease that, were there to be any further canal compromise, might render a patient unsuitable for the planned SSRS, possibly requiring further intervention, i.e., separation surgery first. In such cases it may be more prudent to proceed with radiation therapy first, or if the back pain cannot be medically managed, patients may benefit from a mechanical posterior tension band such as percutaneous rods and screws that will not affect the spinal canal at the level to be treated. ${ }^{18}$ In the future ablative therapy may play a role in the management of these patients who have associated epidural disease, potentially decreasing tumor volume and counterbalancing the effects of BKP on the spinal canal. ${ }^{20}$

Cruz et al. reported two cases with "tumor extravasation" following BKP that would potentially alter the postkyphoplasty radiation-treatment planning, possibly requiring a change from SSRS to conventional external beam radiation therapy or possibly subjecting the patient to further radiation to cover increased tumor distribution. ${ }^{6}$ The authors described two mechanisms of tumor extravasation. The first proposed mechanism is a pressure effect of the bone tamps displacing tumor through fracture cortex, a hypothesis in part supported by our study, although in our patient cohort there was no evidence of subligamentous tumor displacement either cephalad or caudad to the level that was augmented. In the two case examples reported by Cruz et al., the first follow-up imaging was performed at 4-8 weeks postprocedure, and imaging studies in both cases demonstrated further fracture progression resulting in a loss of vertebral body height, possibly contributing to the subligamentous displacement of tumor. The second mechanism of tumor extravasation was thought to be vascular, resulting from venous extravasation of tumor caused by increased intravertebral pressure associated with bone tamps inflation and/or cement infusion. This venous displacement of tumor was thought to account for adjacent 
level disease as well. This hypothesized mechanism of tumor displacement is not addressed by our paper, as the post-BKP imaging was typically performed within 3 days of the procedure, which is not enough time to identify microscopic vascular displacement of tumor. Additionally, CT myelography is not sensitive enough to detect this mechanism. A recent retrospective review examining the effect of kyphoplasty on local control documented only one local, adjacent level recurrence among 17 patients who had undergone kyphoplasty prior to SSRS. ${ }^{2}$ Disease recurred at 18 months in the setting of marked progression throughout the patient's axial and appendicular skeleton, with the local recurrence thought to be unrelated to the kyphoplasty, neither venous nor mechanical tumor extravasation.

A potential limitation of this study is that two different imaging modalities were used to determine the cross-sectional area of the thecal sac, that is, pre-BKP MR images were compared to post-BKP CT myelograms. Slice thickness is usually greater on MRI than on CT, and volumeaveraging effects can affect determination of the area of the thecal sac. We feel that the ability to reformat the post-BKP axial CT myelograms to closely match the pre-BKP MR images minimized the differences between the two modalities. The time elapsed between pre-BKP MRI and postBKP CT myelography could be a problem if there were disease progression at the levels to be treated. The tumor progression could also contribute to canal compromise on the post-BKP CT scans, though we agreed that there was no definite evidence of disease progression between the prekyphoplasty image and the post-BKP CT myelogram. Fracture progression sometime after the pre-BKP imaging could also result in increased spinal canal compromise secondary to tumor displacement posteriorly into the ventral epidural space. In this study, however, the four vertebrae found to have fracture progression were not associated with further spinal canal compromise. Among the 10 vertebral levels that demonstrated a decreased cross-sectional area of the thecal sac, there was no convincing evidence of fracture progression on the intraoperative fluoroscopic imaging.

\section{Conclusions}

Balloon kyphoplasty can result in increased spinal canal compromise caused by posterior tumor displacement in vertebrae that are associated with destruction of the posterior vertebral body cortex and epidural disease. Although not observed in this cohort of patients, posterior displacement of tumor has significant potential to alter the postkyphoplasty radiation treatment planning and the potential for neurological compromise. Posterior cortex disruption or low-grade epidural spinal cord compression could be considered a potential contraindication to kyphoplasty: however, kyphoplasty prior to SSRS reduces mechanical pain, optimizing patient tolerance to positioning for SSRS and overall pain outcomes without compromising neurological outcomes.

\section{Acknowledgments}

This work was supported in part by NIH/NCI core grant no. P30 CA008748.

\section{References}

1. Aghayev K, Papanastassiou ID, Vrionis F: Role of vertebral augmentation procedures in the management of vertebral compression fractures in cancer patients. Curr Opin Support Palliat Care 5:222-226, 2011

2. Barzilai O, DiStefano N, Lis E, Yamada Y, Lovelock DM, Fontanella AN, et al: Safety and utility of kyphoplasty prior to spine stereotactic radiosurgery for metastatic tumors: a clinical and dosimetric analysis. J Neurosurg Spine 28:7278,2018

3. Bate BG, Khan NR, Kimball BY, Gabrick K, Weaver J: Stereotactic radiosurgery for spinal metastases with or without separation surgery. J Neurosurg Spine 22:409-415, 2015

4. Berenson J, Pflugmacher R, Jarzem P, Zonder J, Schechtman K, Tillman JB, et al: Balloon kyphoplasty versus non-surgical fracture management for treatment of painful vertebral body compression fractures in patients with cancer: a multicentre, randomised controlled trial. Lancet Oncol 12:225-235, 2011

5. Bilsky MH, Laufer I, Fourney DR, Groff M, Schmidt MH, Varga PP, et al: Reliability analysis of the epidural spinal cord compression scale. J Neurosurg Spine 13:324-328, 2010

6. Cruz JP, Sahgal A, Whyne C, Fehlings MG, Smith R: Tumor extravasation following a cement augmentation procedure for vertebral compression fracture in metastatic spinal disease. J Neurosurg Spine 21:372-377, 2014

7. Dublin AB, Hartman J, Latchaw RE, Hald JK, Reid MH: The vertebral body fracture in osteoporosis: restoration of height using percutaneous vertebroplasty. AJNR Am J Neuroradiol 26:489-492, 2005

8. Esmende SM, Daniels AH, Palumbo MA: Spinal cord compression after percutaneous kyphoplasty for metastatic compression fracture. Spine J 13:831-832, 2013

9. Fourney DR: Vertebroplasty versus kyphoplasty in the cancer setting: rethinking the relative indications. Support Cancer Ther 3:26-27, 2005

10. Garfin SR, Yuan HA, Reiley MA: New technologies in spine: kyphoplasty and vertebroplasty for the treatment of painful osteoporotic compression fractures. Spine (Phila Pa 1976) 26:1511-1515, 2001

11. Gerszten PC, Burton SA, Ozhasoglu C, Welch WC: Radiosurgery for spinal metastases: clinical experience in 500 cases from a single institution. Spine (Phila Pa 1976) 32:193-199, 2007

12. Gerszten PC, Germanwala A, Burton SA, Welch WC, Ozhasoglu C, Vogel WJ: Combination kyphoplasty and spinal radiosurgery: a new treatment paradigm for pathological fractures. J Neurosurg Spine 3:296-301, 2005

13. Hentschel SJ, Burton AW, Fourney DR, Rhines LD, Mendel E: Percutaneous vertebroplasty and kyphoplasty performed at a cancer center: refuting proposed contraindications. J Neurosurg Spine 2:436-440, 2005

14. Husain ZA, Sahgal A, De Salles A, Funaro M, Glover J, Hayashi M, et al: Stereotactic body radiotherapy for de novo spinal metastases: systematic review. J Neurosurg Spine 27:295-302, 2017

15. Kaloostian PE, Yurter A, Zadnik PL, Sciubba DM, Gokaslan ZL: Current paradigms for metastatic spinal disease: an evidence-based review. Ann Surg Oncol 21:248-262, 2014

16. Lieberman IH, Dudeney S, Reinhardt MK, Bell G: Initial outcome and efficacy of "kyphoplasty" in the treatment of painful osteoporotic vertebral compression fractures. Spine (Phila Pa 1976) 26:1631-1638, 2001

17. Moussazadeh N, Lis E, Katsoulakis E, Kahn S, Svoboda M, DiStefano NM, et al: Five-year outcomes of high-dose singlefraction spinal stereotactic radiosurgery. Int J Radiat Oncol Biol Phys 93:361-367, 2015

18. Moussazadeh N, Rubin DG, McLaughlin L, Lis E, Bilsky 
MH, Laufer I: Short-segment percutaneous pedicle screw fixation with cement augmentation for tumor-induced spinal instability. Spine J 15:1609-1617, 2015

19. Ortiz Gómez JA: The incidence of vertebral body metastases. Int Orthop 19:309-311, 1995

20. Tatsui CE, Stafford RJ, Li J, Sellin JN, Amini B, Rao G, et al: Utilization of laser interstitial thermotherapy guided by real-time thermal MRI as an alternative to separation surgery in the management of spinal metastasis. J Neurosurg Spine 23:400-411, 2015

21. Wardlaw D, Cummings SR, Van Meirhaeghe J, Bastian L, Tillman JB, Ranstam J, et al: Efficacy and safety of balloon kyphoplasty compared with non-surgical care for vertebral compression fracture (FREE): a randomised controlled trial. Lancet 373:1016-1024, 2009

\section{Disclosures}

Dr. Lis has received compensation from Medtronic for educational teaching. Dr. Laufer has been a consultant for Globus,
Medtronic, DePuy/Synthes, Spinewave, and Brainlab. Dr. Yamada has received compensation from Varian Medical Systems, Brainlab, and Vision RT as part of their speakers bureau and has served on the medical advisory board for the Chordoma Foundation.

\section{Author Contributions}

Conception and design: Lis, Bilsky. Acquisition of data: Lis, Laufer, Yamada, Karimi. Analysis and interpretation of data: Lis, Bilsky. Drafting the article: Lis. Critically revising the article: Lis, Bilsky. Reviewed submitted version of manuscript: Laufer, Barzilai, Yamada, Karimi, McLaughlin, Krol, Bilsky. Administrative/ technical/material support: McLaughlin. Study supervision: Lis, Bilsky.

\section{Correspondence}

Eric Lis: Memorial Sloan Kettering Cancer Center, New York, NY. lise@mskcc.org. 